\title{
Predominance of CTX-M-15 among ESBL Producers from Environment and Fish Gut from the Shores of Lake Victoria in Mwanza, Tanzania
}

\author{
Nyambura Moremi ${ }^{1}$, Elizabeth V. Manda ${ }^{1}$, Linda Falgenhauer ${ }^{2,3}$, Hiren Ghosh ${ }^{2,3}$, \\ Can Imirzalioglu ${ }^{2,3}$, Mecky Matee $^{4}$, Trinad Chakraborty ${ }^{2,3}$ and Stephen E. Mshana ${ }^{1 *}$ \\ ${ }^{1}$ Department of Microbiology and Immunology, Weill Bugando School of Medicine, Mwanza, Tanzania, ${ }^{2}$ Institute of Medical \\ Microbiology, Justus Liebig University, Giessen, Germany, ${ }^{3}$ German Center for Infection Research, Partner site \\ Giessen-Marburg-Langen, Campus Giessen, Giessen, Germany, ${ }^{4}$ Department of Microbiology/Immunology, Muhimbill \\ University of Health and Allied Sciences, Dar es Salaam, Tanzania
}

\section{OPEN ACCESS}

Edited by:

Benoit Doublet,

National Institute for Agricultural

Research, France

Reviewed by:

Nitton Lincopan,

University of São Paulo, Brazil

Andrea Endimiani,

University of Bern, Switzerland

*Correspondence:

Stephen E. Mshana

stephen72mshana@gmail.com

Specialty section:

This article was submitted to Antimicrobials, Resistance and

Chemotherapy,

a section of the journal

Frontiers in Microbiology

Received: 13 July 2016 Accepted: 04 November 2016 Published: 01 December 2016

Citation:

Moremi N, Manda EV, Falgenhauer L,

Ghosh H, Imirzalioglu C, Matee M, Chakraborty T and Mshana SE (2016)

Predominance of CTX-M-15 among

ESBL Producers from Environment and Fish Gut from the Shores of Lake

Victoria in Mwanza, Tanzania.

Front. Microbiol. 7:1862.

doi: 10.3389/fmicb.2016.01862
Extended-Spectrum Beta-Lactamase (ESBL)-producing bacteria are a common cause of healthcare and community-associated infections worldwide. The distribution of such isolates in the environment and their presence in fish as a result of sewage contamination is not well-studied. Here we examined fish and environmental samples from Mwanza city for the presence of ESBL-producing bacteria. From 196 fish sampled from local markets, 26 (13.3\%) contained lactose-fermenting ESBL-producing bacteria, while 39/73 (53.4\%) environmental samples from the same area were ESBL producers. Antibiotic resistance genes, multi locus sequence types (MLST) and plasmid replicon types in 24 selected isolates from both populations were identified with whole genome sequencing using Illumina MiSeq. Nine of eleven sequenced fish isolates had the blaCTX-M-15 gene whereas 12/13 from environment carried bla resistance to sulfonamides (sul1/sul2), tetracyclines [tet(A)/tet(B)] fluoroquinolones [e.g., aac(6')-Ib-cr, qnrS1], aminoglycosides [e.g., aac(3)-IId, strB, strA,] and trimethoprim (e.g., dfrA14) were detected. E. coli sequence type ST-38 (2) and ST-5173 (2) were detected in isolates both from the environment and fish. IncY plasmids carrying bla strA, and strB were detected in five environmental $E$. coli isolates and in one $E$. coli isolate from fish. Our data indicate spillage of resistant environmental isolates into Lake Victoria through the sewage system. Persistence of bla $\mathrm{A}_{\mathrm{CTX}-\mathrm{M}-15}$ in the Mwanza city environment is complex, and involves both clonal spread of resistant strains as well as dissemination by commonly occurring IncY plasmids circulating in isolates present in humans, the environment as well as in the food chain.

Keywords: bla $\mathrm{CTX}$-M-15, fish, environment

\section{INTRODUCTION}

The extended-spectrum beta-lactamases (ESBL)-producing Gram-negative bacteria Escherichia coli and Klebsiella spp., particularly those producing CTX-M enzymes, have emerged as important causative agents of healthcare-associated infections across the world (Hawkey and Jones, 2009). Apart from being present in hospitals and clinics, ESBL-producing E. coli strains are prevalent in 
the community and are reported to be responsible for community-acquired bacterial infections (Arpin et al., 2005; Pitout et al., 2005). Studies from different areas of the world show an association between the presence of ESBL-producing E. coli and exposure to either food or contaminated water (Ho et al., 2011; Laube et al., 2013; Xi et al., 2015). The presence of ESBL-producing $E$. coli in the community has led to the hypothesis that there could be a transmission of these strains from human waste to the environment (Martinez, 2009a). Evidence in the literature has also documented the probable horizontal transfer of resistance genes from either human sewage or clinical isolates to fish in rivers or lakes in which drainage of wastewater from treatment plants occurs (Kümmerer, 2009; Martinez, 2009b; Jiang et al., 2012; Blaak et al., 2014). Multiple genotypes of ESBL-producing E. coli have been found in animals and humans in Mwanza (Mshana et al., 2011, 2016; Seni et al., 2016). There are few studies from developed countries on the presence of ESBL-producing isolates in fish and environment samples and the role played by the food chain in transmission of resistance genes through contamination by human and animals sewage and anthropogenic activities in relation to water bodies (Zurfluh et al., 2013; Abgottspon et al., 2014). In Mwanza, a port city with a population of 750,000 located on the southern shores of Lake Victoria (Fitzpatrick et al., 2015), effluents from wastewater treatment plants and pit-latrines used by most of households located in the hills drain into the lake, which is also a source of fish consumed by residents as staple food. We previously used conventional phenotypic characterization and whole genome sequencing to examine samples obtained from hospitals, from rural farming communities including animals, farmers and households, in Mwanza for the presence of ESBLproducing Enterobacteriaceae. Our data indicate clonal spread of bacteria belonging to a small number of STs present in all populations investigated, but also suggest that commonly occurring promiscuous plasmids are involved in resistance dissemination (Fortini et al., 2015; Mshana et al., 2016). Here we examined for the presence of ESBL-producing bacteria in fish obtained from Lake Victoria as well as environmental samples obtained from the city. Our results indicate that E. coli genotypes that were observed in humans and animals before are also present in environment and Fish.

\section{MATERIALS AND METHODS}

\section{Isolation and Identification of Bacterial Isolates}

Ten fish markets located in different urban and rural sections of the Mwanza region were randomly selected for the study. These markets receive fishes obtained from different fishing sites within Lake Victoria. A total of 196 Nile tilapia (Oreochromis niloticus) fish from randomly picked vendors were sampled with an average of 15 to 20 fish at each market between July and September 2015. Fish were first washed with saline, following which a sterile surgical blade was used to open the carcass and to make a longitudinal incision along the gut. The incision was opened and the gut contents were swabbed using a sterile swab.
In addition, at ten sites including the Ngerengere River that crosses the city, drain waste and possible sewage from households located in the surrounding hills were sampled (environmental samples). Environmental samples included dirty muddy water samples from different location in the city (Figure 1). For each site between six to seven samples were taken from different locations (Figure 1). About $3 \mathrm{ml}$ of each sample was collected using sterile $25 \mathrm{ml}$ falcon tube (BD, Nairobi, Kenya). A sample was mixed with sterile $0.9 \%$ saline at ratio of $1: 1$ and vortexed to produce a homogenous solution.

Using swabs, MacConkey agar (Oxoid, Basingstoke, UK) plates supplemented with $2 \mathrm{mg} / \mathrm{L}$ cefotaxime (Medochemie Ltd, Limassol, Cyprus) were inoculated to screen for ESBL-producing Enterobacteriaceae. Enteric bacilli were identified using colony morphology and differentiated based on lactose fermentation on MacConkey agar. Single colonies from predominant lactose fermenting bacteria were picked for further identification using several biochemical tests (Triple Sugar Iron Agar, Simmons' citrate Agar, Sulfur-Indole-Motility test and Urease test) (Murray et al., 1995). In case of ambiguous results, the VITEK $^{\circledR} 2$ system (BioMérieux, Marcy l'Etoile, France) was used to confirm identification. A confirmed ESBL-producing E. coli isolate was used as a positive and E. coli ATCC 25922 as a negative control. ESBL isolates were stored as glycerol cultures at $-80^{\circ} \mathrm{C}$ and used for further characterization.

\section{Antimicrobial Susceptibility Testing}

Antimicrobial susceptibility testing was done by disk diffusion method as recommended by the Clinical Laboratory Standards Institute (CLSI) guidelines (Wayne, 2012). A bacterial suspension of $0.50 \mathrm{McF}$ arland standard turbidity was prepared from pure culture. An inoculum was then plated on Mueller-Hinton Agar plates (HiMedia, Mumbai, India) and the following antibiotic disks were set: Tetracycline $(2 \mu \mathrm{g})$, ciprofloxacin (5 $\mu \mathrm{g})$, gentamicin $(10 \mu \mathrm{g})$, or trimethoprim/sulphamethoxazole $(1.25 / 23.75 \mu \mathrm{g})$ (Oxoid, Hampshire, UK). The plates were incubated aerobically at $37^{\circ} \mathrm{C}$ for $18-24 \mathrm{~h}$. The diameters of the respective zone of inhibitions were measured and interpreted following CLSI 2012 guidelines (Wayne, 2012). Disk approximation method based on CLSI guidelines was used to confirm ESBL production and for selected isolates ESBL production was further identified using VITEK ${ }^{\circledR} 2$ system (BioMérieux, Marcy l'Etoile, France) and in addition the MIC for cefepime, carbapenems and colistin were determined in all selected isolates.

Using STATA version 11 the two-sample test of proportion was done to compare the rates of resistance between ESBL isolates from fish and those from environment. A $p$-value $<0.05$ was used to indicate a significant difference at $95 \%$ confidence interval.

\section{Whole Genome Sequencing}

Eleven ESBL-producing isolates obtained from fish and $13 \mathrm{E}$. coli from the environmental samples were chosen for whole genome sequencing (WGS). DNA was isolated using the Purelink Genome DNA Mini kit (Invitrogen, Darmstadt, Germany) according to the manufacturer's instruction. WGS was carried 


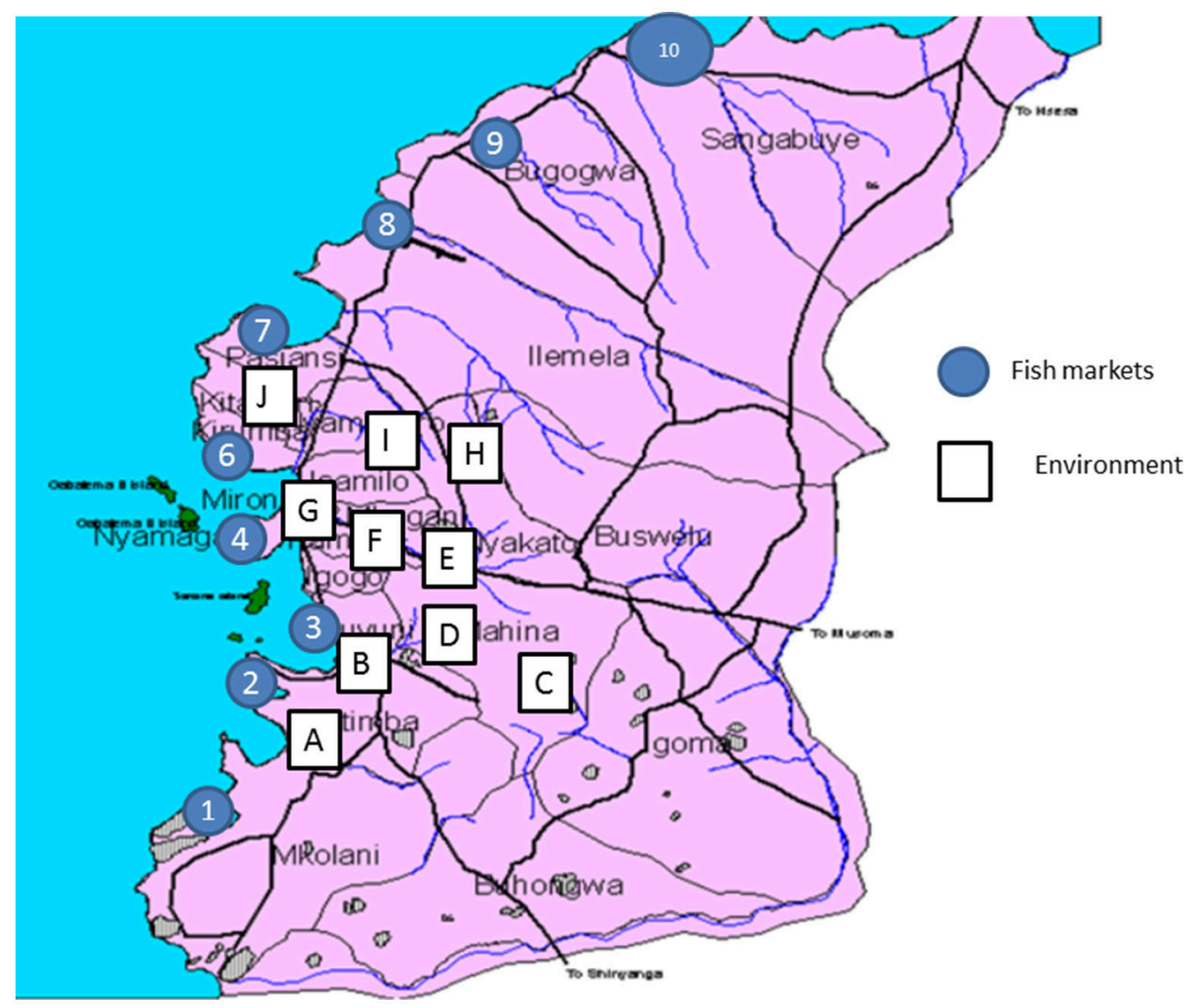

FIGURE 1 | Map of Mwanza City showing fish markets and sites from which environmental samples were obtained.

out using an Illumina Nextera XT library with 2x300bp pairedend reads on an Illumina MiSeq instrument (Illumina, San Diego, CA, USA). The raw data was assembled using SPAdes (version 3.0) (Bankevich et al., 2012). Contigs from E. coli isolates were ordered by using MAUVE (Rissman et al., 2009) and E. coli MG1655 (accession number U00096.3) were chosen as a reference for all E. coli isolates, Citrobacter freundii strain P10159 for Citrobacter braakii isolates (accession number CP012554; no complete genome available for C. braakii), Klebsiella pneumoniae strain ATCC BAA-2146 (accession number CP006659) for $K$. pneumoniae and Enterobacter cloacae strain 34977 (accession number CP010376) for E. cloacae isolates. Pseudogenomes were created and whole genome phylogenetic analysis was subsequently performed by using ParSNP package of Harvest Suite (Treangen et al., 2014). The raw sequencing data of the sequenced isolates are available at the European Nucleotide Archive (ENA) under the project number PRJEB12361.

\section{In silico Analyses of Resistance Genes, MLST, Plasmid Replicon Types, and Quinolone Resistance-Determining Regions (QRDR) Mutations}

Sequences were analyzed for their multi locus sequence types, transferrable resistance genes, plasmid replicon types and pMLST using MLST 1.8, ResFinder, Plasmidfinder and pMLST software from the Center for Genomic Epidemiology (Larsen et al., 2012; Zankari et al., 2012; Carattoli et al., 2014). Search for plasmid-encoded heavy metal resistances and detergence resistance was performed using blastn with the references given in Supplementary Table 1.

The location of bla $a_{\mathrm{CTX}-\mathrm{M}-15}$ was determined by analyzing the contigs harboring bla $a_{\mathrm{CTX}-\mathrm{M}-15}$ using blastn. The whole genome sequences were compared with the plasmid pPGRT46 (accession number KM023153.1) using BRIG and blastn (Alikhan et al., 2011; Fortini et al., 2015). Quinolone resistance-determining regions (QRDR) mutations were identified by silico analysis by comparing the sequence using a reference sequence from a quinolone-susceptible Enterobacteriaceae strain (Weigel et al., 1998; Liu et al., 2012).

\section{Ethical Approval}

The protocol of this study was approved by CUHAS/BMC joint ethics and scientific review committee with reference CREC/019/2014.

\section{RESULTS}

\section{Bacterial Isolates}

A total of $26(13.3 \%)$ lactose-fermenting ESBL-producing bacteria were isolated from gut samples of 196 wild Nile tilapia fish from Lake Victoria. Diverse bacterial species were 
detected and included C. braakii (11/26, 42.3\%), E. cloacae (5/26, $19.2 \%)$, K. pneumoniae $(5 / 26,19.2 \%)$, E. coli $(4 / 26,15.4 \%)$ and Klebsiella oxytoca $(1 / 26,3.9 \%)$. From 73 environmental samples 39 (53.4\%) ESBL-producing enteric bacteria were isolated. Of these 39 isolates, $20(51.3 \%)$ were E. coli and 19 (48.7\%) were K. pneumoniae.

\section{Antimicrobial Susceptibility Testing}

All isolates had MICs for cefotaxime and cefepime of $>32$ and $>8 \mu \mathrm{g} / \mathrm{ml}$ respectively. High resistance rates to co-trimoxazole ( $n=19,73.1 \%)$, ciprofloxacin $(n=19,73.1 \%)$, and gentamicin $(n=19,73.1 \%)$ and tetracycline $(n=16,61.5 \%)$ were observed among the $26 \mathrm{ESBL}$-producing isolates from fish. Environmental isolates were resistant to co-trimoxazole ( $n=34,87.2 \%)$, ciprofloxacin ( $n=14,38 \%)$, and gentamicin ( $n=18,46.1 \%)$. ESBL-producing isolates from fish were significantly more resistant to gentamicin and ciprofloxacin $(p<0.01$ : Table 1$)$. In addition, all isolates were sensitive to meropenem and imipenem $(\mathrm{MIC}<0.25 \mu \mathrm{g} / \mathrm{ml})$, and all were sensitive to colistin.

\section{Analysis of Antibiotic Resistance Genes}

Each of the bacterial genome-sequenced fish and environmental isolates carried up to four different $\beta$-lactamase genes (ESBL, AmpC and other $\beta$-lactamases, Table 2). The most common

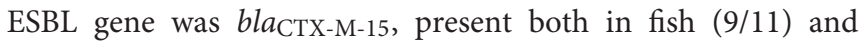
environmental isolates (12/13). The non-ESBL $\beta$-lactamase gene bla $a_{\text {TEM-1 }}$ was present in 7/11 fish isolates and 9/13 environmental isolates, whereas bla $a_{\mathrm{OXA}-1}$ was present in 6/11 fish isolates and $1 / 13$ environmental isolates. K. pneumoniae isolates from fish were found to harbor bla $a_{\mathrm{SHV}-11}$ and bla $a_{\mathrm{SHV}-1}$ due to the fact that K. pneumoniae chromosomally possesses bla $a_{\mathrm{SHV}-1 /-11}$. In addition, the fish isolates also harbored a number of AmpC type $\beta$-lactamase genes (bla $\left.a_{\mathrm{ACT}-15}, b l a_{\mathrm{MIR}-3}, b l a_{\mathrm{CMY}-37}, b l a_{\mathrm{CMY}-49}\right)$, which were not present in the environmental isolates. The latter two results might simply reflect the fact that while different types of enterobacterial species were isolated from fish, only E. coli were obtained from the environmental samples studied.

Commonly occurring aminoglycoside resistance genes detected in both fish and environmental isolates were $a a c\left(6^{\prime}\right)$ Ib-cr (6/24), strA/strB (15/24), aac(3)-IId (5/24), and aadA1 (4/24) (Table 2). Other aminoglycoside resistance genes were present either only in fish isolates (aadA2, aac(3)-IIa) or in environmental samples (aadA5). Quinolone resistance genes were detected in 8/11 (72.7\%) of fish isolates and in 7/13 (53.7\%)

TABLE 1 | Rate of resistance of ESBL-producing isolates from fish and environment to SXT, TET, CIP, and CN.

\begin{tabular}{|c|c|c|c|}
\hline Antibiotic & ESBL fish (26) & ESBL environment (39) & $P$-value \\
\hline SXT & 19 (73\%, 95\% Cl 55.9-90.6) & 34 (87.2\%, 95\% Cl 76-97) & 0.0776 \\
\hline TET & 17 (65.4\%, 95\% Cl 47-83) & $27(69.2 \%, 95 \%$ Cl 54.7-83.6) & 0.3741 \\
\hline CIP & 19 (73\%, 95\% Cl 55.9-90.6) & 15 (38.5\%, 95\% Cl 23-53) & 0.0032 \\
\hline $\mathrm{CN}$ & 19 (73\%, 95\% Cl 55.9-90.6) & $18(46.1 \%, 95 \%$ Cl 37-50 & 0.001 \\
\hline
\end{tabular}

of environmental isolates that were sequenced. Quinolone resistance genes detected in both populations comprised of the $a a c\left(6^{\prime}\right)-I b-c r(6 / 24), q n r \mathrm{~B} 1(5 / 24)$, and $q n r S 1$ alleles. Other quinolone resistance genes were only present in fish isolates ( $q n r \mathrm{~B} 29, q n r \mathrm{~B} 48$, oq $x \mathrm{~A}$, oq $x \mathrm{~B})$.

Of the 24 sequenced isolates, 20 (83.3\%) harbored sulfonamide resistance genes (sul1 or sul2), and 19 of these isolates harbored in addition a resistance gene encoding trimethoprim resistance ( $d$ frA $14, n=11 ; d f r A 17, n=3$; $d f r A 1, d f r A 18, d f r A 30, d f r A 5, d f r A 7$, each $n=1)$. Tetracycline resistance genes, [tet $(A), n=15 ; \operatorname{tet}(D), n=3]$, were present in $18 / 24$ isolates. Altogether, there was a direct correlation between resistance phenotype and resistance genotype in all 24 isolates.

Using in silico analysis parC mutations were detected in 7 E. coli, only 2 isolates had S80I mutation that is associated with fluoroquinolone resistance, while gyr A mutations were observed in 9 isolates (2 E. cloacae and 7 E. coli). S83 (S83T, S83L, and $\mathrm{S} 83 \mathrm{~A}$ ) mutations that are associated with fluoroquinolone resistance were observed in 5/9 isolates. One isolate had both S83L and D87N.

\section{Plasmid-Encoded Heavy Metal Resistance Operons and Detergence Genes}

An analysis for the presence of plasmid-located heavy metal- and detergent- resistance genes revealed, that $8 / 24$ isolates harbored the qacEdelta gene, conferring resistance to tertiary ammonium compounds. Seven (29.2\%) of isolates sequenced carried a mercury resistance operon originally described in plasmid R478, and $4 / 24(16.6 \%)$ isolates had genes mediating resistance to silver. Only one isolate carried genes involved in resistance to copper together with nickel/cobalt efflux system (Supplementary Table 2).

\section{Location of the bla Genes}

Analysis of the whole genome sequences was used to identify and map the location of bla CTX-M alleles. For 8/24 isolates,

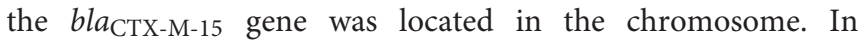
two isolates bla $a_{\text {CTX-M-15 }}$ was located on a phage-like plasmid, similar to the E. coli phage-like plasmid p1303_95 isolated from wound swab (isolate SO007, accession number of the reference: CP009168.1) and pECOH89 (isolate SO053, accession number of pECOH89: HG530657.1) (Falgenhauer et al., 2014). The isolate SO069 harbored bla $a_{\mathrm{CTX}-\mathrm{M}-15}$ on an IncI1 plasmid similar to pESBL-EA11 (Ahmed et al., 2012). Six of 24 sequenced isolates (one fish and five environmental isolates, F044, SO005, SO008, SO025, SO037, SO063, all E. coli) harbored a resistance cassette similar to the one present in plasmid pPGRT46 (accession number KM023153.1) that included both $q n r S 1$ as well as bla $a_{\mathrm{CTX}-\mathrm{M}-15}$. The other isolates displayed bla $a_{\mathrm{CTX}-\mathrm{M}-15}$ or bla $a_{\mathrm{CTX}-\mathrm{M}-55}$ containing resistance cassettes with similarities to other ESBL-encoding plasmids (F025: pEC_L46, accession number GU371929.1; F102: pSTm-A54650, accession number LK056646.1; SO042: pSKLX3330, accession number KJ866866.1; SO060: pCA14, accession number CP009231.1). The isolates F016 and F017 did not harbor any ESBL 


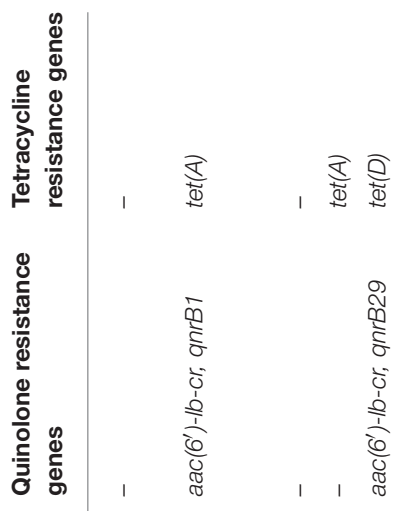

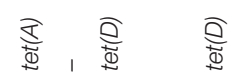

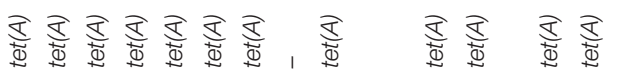

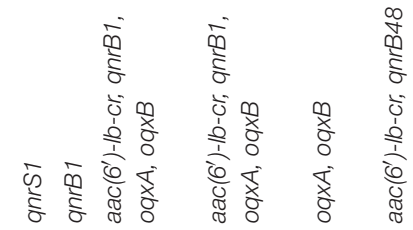

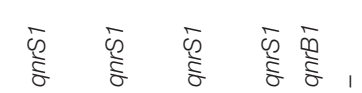

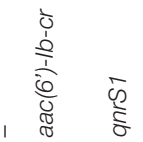

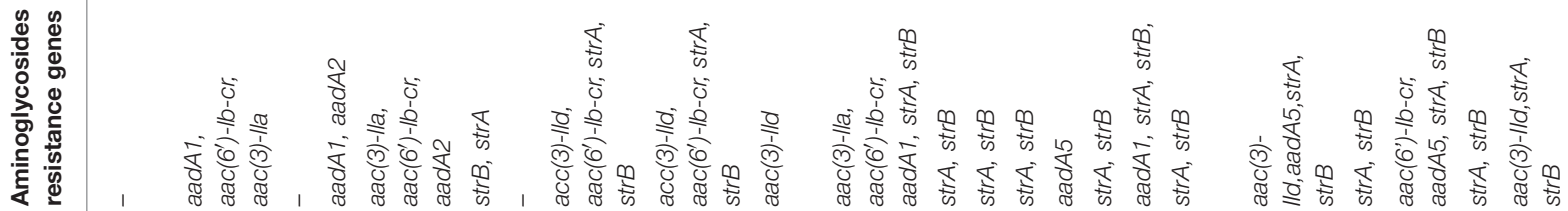

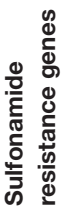

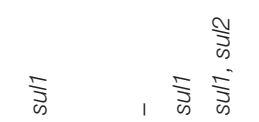

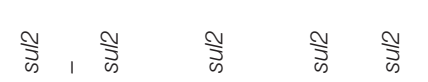

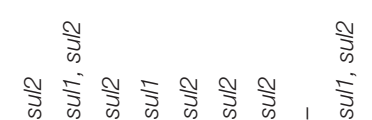

$\stackrel{2}{\cong}$

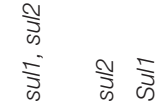

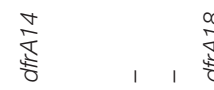

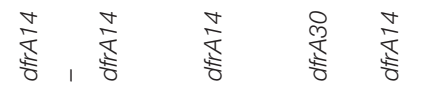

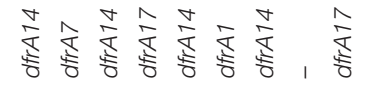

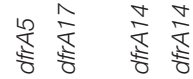

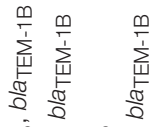
Q

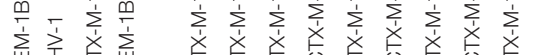

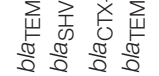

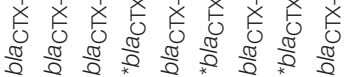

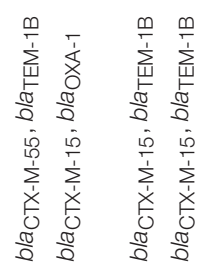

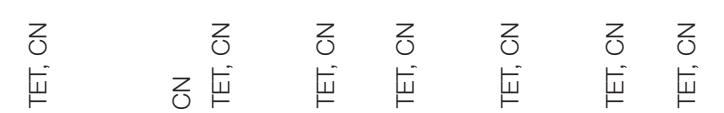

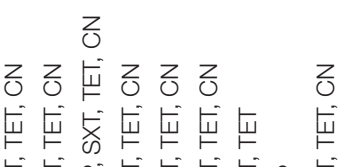

z

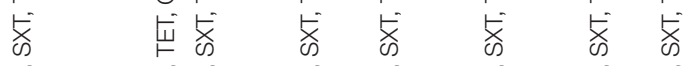

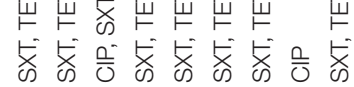

$\stackrel{\uplus}{\uplus}$

$\frac{0}{0} \quad \frac{0}{0} \quad \frac{0}{0} \quad \frac{0}{0} \quad \frac{0}{0} \quad \frac{0}{0} \quad \frac{0}{0}$

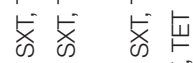

远 这
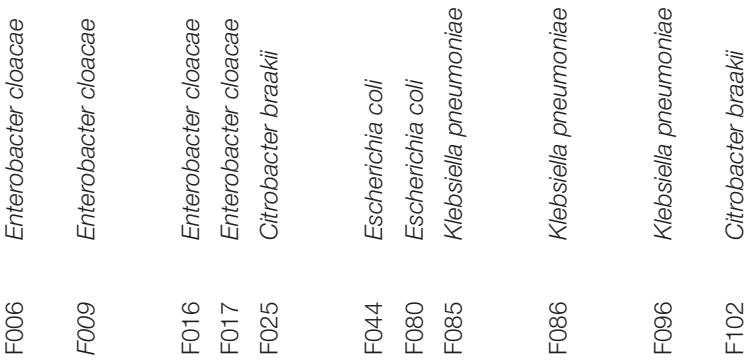

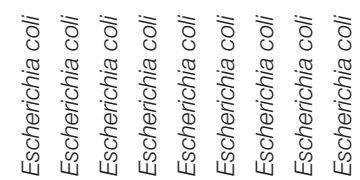

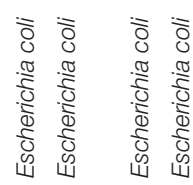

喜兽器

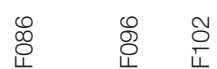

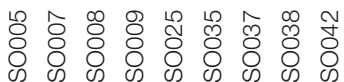

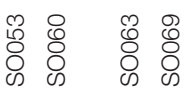


resistance gene. It should be noted that because conjugation experiments were not done plasmid location could only be suggested.

In 13 of 22 ESBL-encoding isolates, the environment of the ESBL gene was characterized by a Tn3 transposon deletion in the vicinity of the bla $a_{\mathrm{CTX}-\mathrm{M}}$ gene (Figure 2). This included four isolates harboring a chromosomally located bla allele. The other isolates, including two chromosomally located bla $a_{\text {CTX-M-15 }}$ and the single bla $a_{\text {CTX-M-55 }}$ isolate, did not have any Tn3-related sequences. All ampC genes detected were located in the chromosomes (F006, F009, F016, F025, F102).

\section{Multi Locus Sequence Types and Whole Genome Phylogeny}

E. coli isolates sequence type (ST) ST-38 and ST-5173 were present in both fish and environmental isolates. Among the 13 E. coli environmental isolates 12 different STs were observed. The fifteen $E$. coli isolates, 13 from the environment and 2 from fish, grouped into five clusters using genome-based phylogenetic analysis. The tree was generated on the basis of a core genome accounting for $78 \%$ (3.62 Mbp) of the reference genome (E. coli MG1655). The two E. coli isolates from fish have $100 \%$ similarity with respective ST isolates derived from the environment (SO025 and F044, ST-38; F080 and SO038, ST-5137; Figure 3). E. cloacae

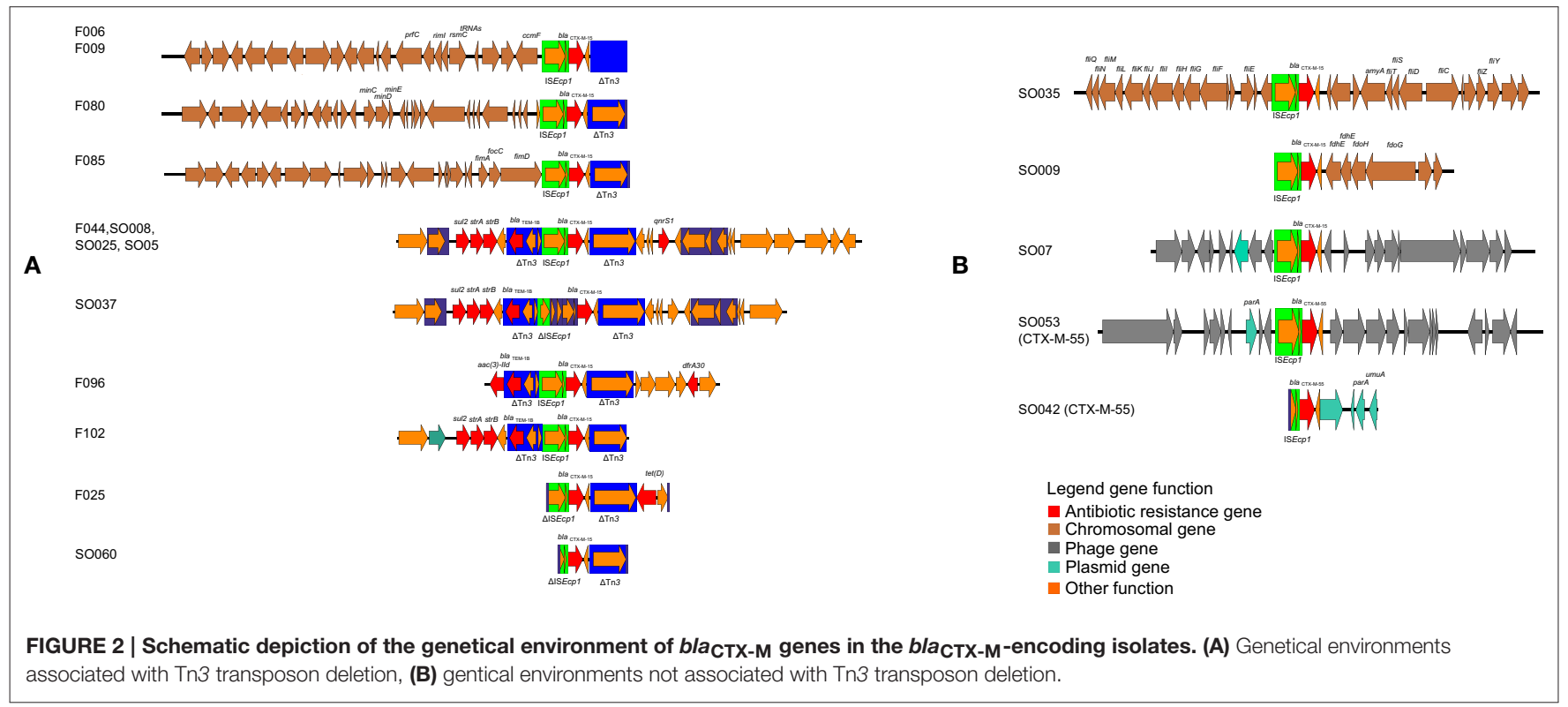

A

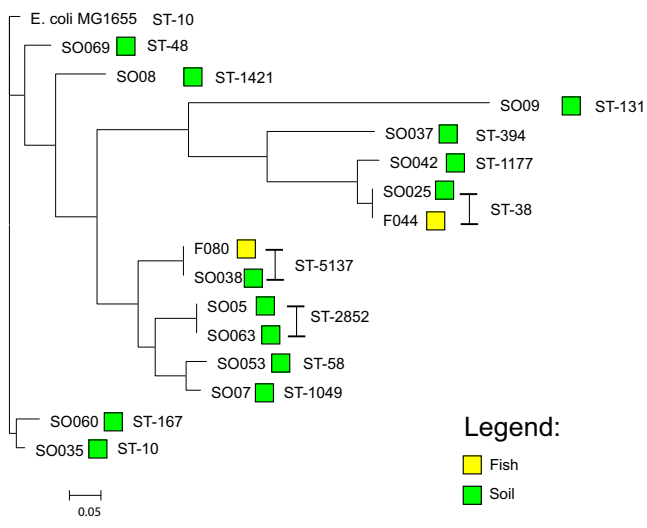

B

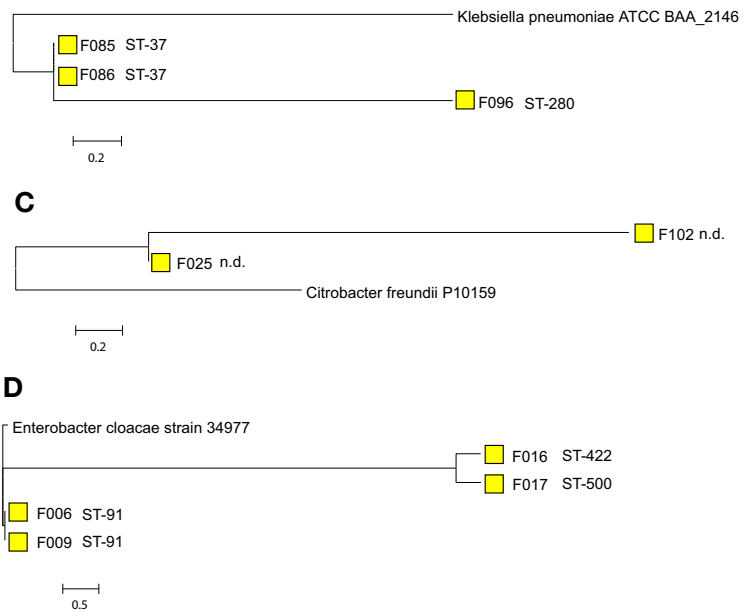

FIGURE 3 | Core-genome based phylogeny of the sequenced isolates according to their species (A) Escherichia coli (reference E. coli MG1655, accession number U00096.3), (B) Klebsiella pneumoniae (reference Klebsiella pneumoniae strain ATCC BAA-2146, accession number CP006659), (C) Citrobacter braakii (using Citrobacter freundii P10159, accession number CP012554.1 as a reference as no complete genome of Citrobacter braakii is presently available), (D) Enterobacter cloacae (E. cloacae strain 34977, accession number CP010376). The phylogenetic analysis was performed using Harvest Suite. 
TABLE 3 | Baseline characteristics of the sequenced isolates.

\begin{tabular}{lllll}
\hline No & Species & Sequence type & Phylogroups & Source \\
\hline F006 & Enterobacter cloacae & ST-91 & & Fish \\
F009 & Enterobacter cloacae & ST-91 & & Fish \\
F016 & Enterobacter cloacae & ST-422 & & Fish \\
F017 & Enterobacter cloacae & ST 500 & & Fish \\
F025 & Citrobacter braakii & $*$ & & Fish \\
F044 & Escherichia coli & ST-38 & E & Fish \\
F080 & Escherichia coli & ST-5173 & B1 & Fish \\
F085 & Klebsiella pneumoniae & ST-37 & & Fish \\
F086 & Klebsiella pneumoniae & ST-37 & & Fish \\
F096 & Klebsiella pneumoniae & ST-280 & & Fish \\
F102 & Citrobacter braakii & $*$ & & Fish \\
S0005 & Escherichia coli & ST-2852 & B1 & Environment \\
S0007 & Escherichia coli & ST-1049 & B1 & Environment \\
S0008 & Escherichia coli & ST-1421 & A & Environment \\
S0009 & Escherichia coli & ST-131 & B2 & Environment \\
SO025 & Escherichia coli & ST-38 & E & Environment \\
SO035 & Escherichia coli & ST-10 & E & Environment \\
SO037 & Escherichia coli & ST-394 & E & Environment \\
SO038 & Escherichia coli & ST-5173 & B1 & Environment \\
S0042 & Escherichia coli & ST-1177 & E & Environment \\
SO053 & Escherichia coli & ST-58 & B1 & Environment \\
S0060 & Escherichia coli & ST-167 & E & Environment \\
S0063 & Escherichia coli & ST-2852 & B1 & Environment \\
S0069 & Escherichia coli & ST-48 & E & Environment \\
\hline
\end{tabular}

"There is no MLST scheme available for Citrobacter braakii.

isolates from fish displayed three different STs (ST-91 $n=2$; ST-422 $n=1$; ST-500 $n=1$ ), based on an analysis of core genome content of around 68\% (3.33 Mbp) using E. cloacae strain 34977 as reference. The two ST-91 isolates were more closely related to each other, than the other two isolates. K. pneumoniae isolates were typed as ST-37 (2/3) with a single isolate as ST$280(1 / 3)$. The core genome with K. pneumoniae strain ATCC BAA-2146 (accession number CP006659) as reference accounted for $88 \%$ (4.78 Mbp) of its genome size. The ST-37 and ST-280 are quite different from one other. The C. braakii isolates could not be assigned to any ST, because there is no typing scheme available. Based on $82 \%$ ( $4.17 \mathrm{Mbp}$ ) core genome size using C. freundii strain P10159 as a reference genome, these two isolates are very distinct from each other (Figure 3). E. coli were grouped in different phylogroups based on scheme of Clermont et al. (2000). A total of 7/15(46\%) of E. coli isolates belong to the newly described phylogroups E (Table 3).

\section{Plasmid Replicon Types}

Of the eleven fish isolates tested, seven (63.6\%) were found to carry IncF plasmid replicon types as compared to $5 / 13$ (38.5\%) of environmental isolates (Table 4). The IncF pMLST types detected in fish isolates were F-: A-:B36, K4:A-:B- and K5:A-:B-. For the environmental isolates IncF pMLST types detected were F2: A:B-, F29:-A-:B10 and F31:A4:B1. The IncI1 plasmids detected in E. coli from environment were classified using pMLST either as
ST-31 and unknown pMLST while that in E. coli from fish had an unknown IncI1 pMLST. Two out of four E. cloacae isolates from fish harbored IncF plasmids with identical pMLST (F-:A:B36). We extracted contig sequences of the F044, SO005, SO008, SO025, SO037, SO063, carrying the resistance cassette present in pPGRT46 (Fortini et al., 2015) (accession no. KM023153) and compared them to this plasmid to examine for overall homology. These isolates showed an overall overlap of between 76 and $90 \%$ in their nucleotide sequences with plasmid pPGRT46 (Figure 4).

\section{DISCUSSION}

Lake Victoria is Africa's largest lake by area, and borders three East-African countries: Tanzania (49\%), Uganda (45\%), and Kenya (6\%). It is not only the major source of fish consumed by Mwanza residents but also receives biologically treated wastewater effluent from Mwanza city, where a tertiary hospital serving eight regions in Tanzania is located. Studies performed in this hospital have shown high rates of ESBL-producing $K$. pneumoniae and E. coli carrying bla $a_{\mathrm{CTX}-\mathrm{M}-15}$ in IncF plasmids (Mshana et al., 2011, 2013). In addition recent studies involving isolates from animals and humans from community have shown the bla ${ }_{\text {CTX-M-15 }}$ allele to be predominant (Mshana et al., 2016; Seni et al., 2016).

In the present study, a significant proportion of fish gut and environmental samples were positive for ESBL-producing bacteria. As previously observed in isolates from humans in hospitals (Mshana et al., 2011, 2013), the majority of these isolates were multiply resistant to co-trimoxazole, gentamicin, tetracycline, and ciprofloxacin. In addition, it was observed that ESBL isolates from fish were significantly more resistant to ciprofloxacin and gentamicin. This could be explained by the fact majority of the environment isolates were E. coli while other genera such as Citrobacter and Enterobacter which tend to be resistant to ciprofloxacin and gentamicin (Dos Santos et al., 2015) formed majority of isolates from Fish. Such high AMR rates detected in the isolates from gut contents of wild fish are of concern and indicate strong anthropogenic environmental contamination of the Lake Victoria. The presence of ESBL isolates in the environmental samples suggests that the Lake Victoria is being contaminated by sewage from the hospitals and from animals (Mshana et al., 2009; Kayange et al., 2010; Moremi et al., 2014; Seni et al., 2016). Farming activities that use of animal manure together with agricultural waste from the community generate steady contaminated effluents along the borders of Lake Victoria. Climatic conditions including sustained periods of rain or man-made erosion may further contribute to increasing the presence of these bacteria in the lake.

The prevalence of ESBL-producing E. coli from wild fish was $2 \%(4 / 196)$ which is comparable to the results previously reported from China (Jiang et al., 2012) but lower than in Egypt which reported a prevalence of $4 \%$ (Ishida et al., 2010). Unlike the two studies from Egypt and China which were conducted among farmed fish with antibiotic exposure, the investigated isolates here were from uncultured free-living fish and emphasize the 
TABLE 4 | Plasmid characteristics of the sequenced isolates, n.t.: not typable using IncHI1 pMLST scheme (no pMLST alleles present); \# variants with homology or coverage less than $100 \%$.

\begin{tabular}{|c|c|c|c|c|c|c|}
\hline No & Species & Plasmid Replicon type & pMLST & ESBL gene & $\begin{array}{l}\text { accession number } \\
\text { of best reference }\end{array}$ & location \\
\hline F006 & Enterobacter cloacae & IncFII, IncFIB & F-:A-:B36\# & bla $\mathrm{CTX}-\mathrm{M}-15$ & CP011650.1 & Chromosome \\
\hline F009 & Enterobacter cloacae & IncFII, IncFIB & F-:A-:B36\# & blacTX-M-15 & СР011650.1 & Chromosome \\
\hline F016 & Enterobacter cloacae & $\operatorname{lncFIB}(K)$ & $\mathrm{F}-: \mathrm{A}-: \mathrm{B}-$ & no ESBL gene & / & / \\
\hline F017 & Enterobacter cloacae & IncFII, IncR & $F-: A-: B-$ & no ESBL gene & / & / \\
\hline F025 & Citrobacter braakii & No replicon & & bla $\mathrm{CTX}-\mathrm{M}-15$ & GU371929.1 & Plasmid \\
\hline F044 & Escherichia coli & Incl1, IncY & 11: new & bla $\mathrm{CTX}-\mathrm{M}-15$ & KM023153.1 & Plasmid \\
\hline F080 & Escherichia coli & No replicon & - & blactX-M-15 & CP011018.1 & Chromosome \\
\hline F085 & Klebsiella pneumoniae & IncFII, IncFIB(K), IncHI1B & F: K4:A-:B-, IncHI1: n.t. & blacTX-M-15 & CP006659.2 & Chromosome \\
\hline F086 & Klebsiella pneumoniae & IncFII, IncFIB(K), IncHI1B & F: K4:A-:B-, IncHl1: n.t. & bla $\mathrm{CTX}-\mathrm{M}-15$ & CP006659.2 & Chromosome \\
\hline F096 & Klebsiella pneumoniae & $\operatorname{lncFII}(\mathrm{K}), \operatorname{IncR}$ & $\mathrm{K} 5: \mathrm{A}-\mathrm{B}-$ & blacTX-M-15 & KM023153.1 & Plasmid \\
\hline F102 & Citrobacter braakii & No replicon & - & bla $\mathrm{CTX}-\mathrm{M}-15$ & LK056646.1 & Plasmid \\
\hline SO005 & Escherichia coli & $\operatorname{IncY}$ & - & blactX-M-15 & KM023153.1 & Plasmid \\
\hline SO007 & Escherichia coli & Incl1, IncP, IncY & 11: new & blactX-M-15 & СР009168.1 & Plasmid \\
\hline SO008 & Escherichia coli & No replicon & - & blacTX-M-15 & KM023153.1 & Plasmid \\
\hline SO009 & Escherichia coli & IncFII, IncFIA & $\mathrm{F} 2: \mathrm{A} 1: \mathrm{B}-$ & bla CTX-M-15 & CP013658.1 & Chromosome \\
\hline SO025 & Escherichia coli & $\operatorname{lncY}$ & - & blacTX-M-15 & KM023153.1 & Plasmid \\
\hline SO035 & Escherichia coli & No replicon & - & blacTX-M-15 & LM995868.1 & Chromosome \\
\hline SO037 & Escherichia coli & IncFII, IncY & - & bla $\mathrm{CTX}-\mathrm{M}-15$ & KM023153.1 & Plasmid \\
\hline S0038 & Escherichia coli & No replicon & - & bla $\mathrm{CTX}-\mathrm{M}-15$ & CP011018.1 & Chromosome \\
\hline SO042 & Escherichia coli & IncFII, IncFIB & F29:A-:B10 & blacTX-M-55 & KJ866866.1 & Plasmid \\
\hline SO053 & Escherichia coli & IncFII, IncFIB, IncQ1 & $\mathrm{F} 2: \mathrm{A}-: \mathrm{B} 1$ & blacTX-M-15 & HG530657.1 & Plasmid \\
\hline SO060 & Escherichia coli & IncFII, IncFIA, IncFIB, IncFII & F31\#:A4:B1 & blacTX-M-15 & СР009231.1 & Plasmid \\
\hline SO063 & Escherichia coli & IncY & - & bla CTX-M-15 & KM023153.1 & Plasmid \\
\hline SO069 & Escherichia coli & Incl1 & I1: ST-31 & bla $\mathrm{CTX}-\mathrm{M}-15$ & СР003290.1 & Plasmid \\
\hline
\end{tabular}

role of environmental pollution with AMR isolates as previously published (Kümmerer, 2009; Guenther et al., 2011).

As was already noted in studies from the Asian, European and the African continents which documented the emergence and

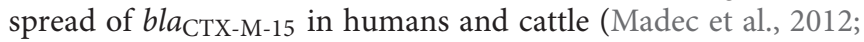
Zhang et al., 2013; Rafaï et al., 2015), CTX-M-15 was also found to be the predominant allele in this study. The gene was located on the chromosome or on different plasmids indicating extreme environmental mobility. The bla $l a_{\mathrm{CTX}-\mathrm{M}-15}$ allele has been reported in other studies (Dobiasova et al., 2015; Fortini et al., 2015; Ibrahim et al., 2015) of Gram-negative bacterial isolates from humans, fish, and animals, suggesting a transmission/circulation of this gene among different settings.

Two plasmid-mediated AmpC enzymes were detected in this study from fish isolates: bla $a_{\mathrm{MIR}-1}$ and $b l a_{\mathrm{ACT}-15}$, this is in contrast with a previous report that showed the presence of plasmid-mediated bla $a_{\mathrm{CMY}-2}$ allele in fish (Welch et al., 2009). No isolates carrying bla $a_{\mathrm{CMY}-2}$ were detected in this study. However, a chromosomally located bla $\mathrm{CMY}-37$ which had previously been described in $C$. freundii, was detected in a C. braakii, (Ahmed and Shimamoto, 2008). One resistant isolate harbored neither a plasmid-based AmpC nor a detectable ESBL enzyme (F017) suggesting the resistance observed might be due to unknown mechanisms or the presence of resistance genes in the chromosome. The chromosomally located genes cannot be detected using the software from the Center for Genomic Epidemiology because the database targets for transferable resistance genes only. As in previous studies performed in China and Egypt among farmed fish (Ishida et al., 2010; Jiang et al., 2012) plasmid-mediated quinolone resistance genes such as $a a c\left(6^{\prime}\right)-I b-c r, q n r \mathrm{~B}$ and $q n r S$ were also detected in this study in both sampled populations.

E. coli ST-38 which was also observed in humans in hospitals, humans in the community and in livestock/ companion/domestic animals in Mwanza city (Mshana et al., 2011, 2016; Seni et al., 2016) was detected in this study in both fish and environmental samples. According to the new phylogroup scheme by Clermont et al. (2000), all these Isolates (human, livestock/companion animal, fish/environment) were grouped as the new phylogroup E emphasizing the uniformity of these strains in our setting. The ST-38 E. coli from fish and the environment as well as other ST types from environment harbor resistance cassettes that include CTX-M-15 and QnrS1. A similar resistance cassette was previously detected on an IncY plasmid detected in isolates from healthy pregnant women in Nigeria, and from ESBL-producing isolates from livestock and companion animal isolates in the Mwanza region (Fortini et al., 2015; Seni et al., 2016). The sequence overlap with the plasmid pPGRT46 was very high (up to 90\%). These findings draw attention to the likelihood that this resistance cassette and its 


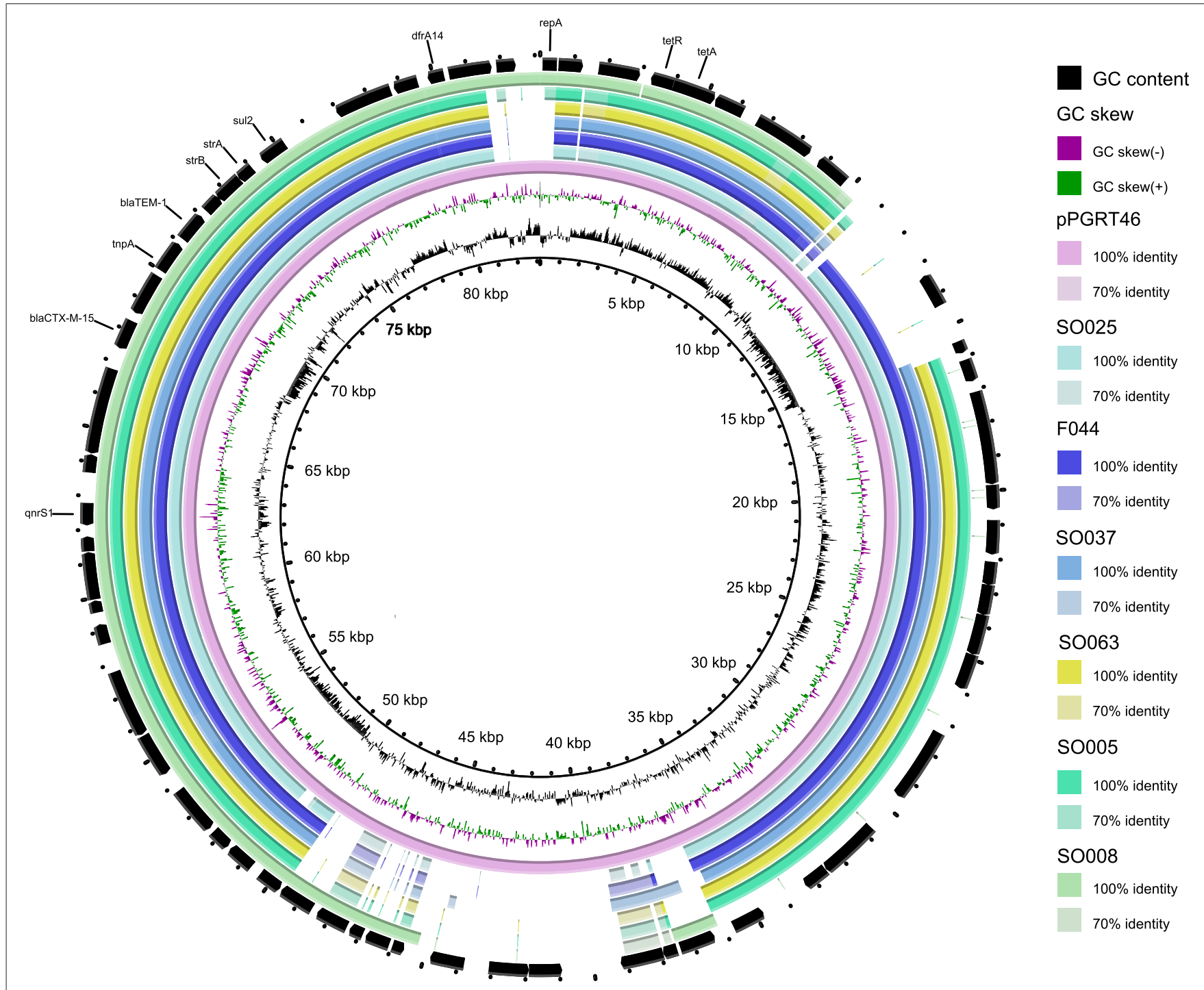

FIGURE 4 | Comparative analysis of the whole genome sequences with plasmid pPGRT46. Alignments were performed using BRIG and depict percent identity between genes from the draft sequences of various plasmids described in this study and the reference plasmid.

plasmid have spread centrally across the African continent into multiple E. coli genotypes and in different environments.

The E. coli ST-38 was observed in both fish and environment isolates as documented above was previously detected in humans and animals in the same setting. This finding is worrisome as zoonotic transmission is possible and its association with human colonization and infection might pose treatment challenges to human health. The transmission and persistence of ESBLproducing bacteria through the food chain and environment through sewage contamination has been documented previously (Beninati et al., 2015; van Hoek et al., 2015). The findings of this study suggest the possibility of the transmission of both ESBL-producing clones and plasmids between humans and wild fish through environmental contamination and indicate that anthropogenic activities and the food chain as potential factors for the persistence and dissemination of bla $a_{\mathrm{CTX}-\mathrm{M}-15}$ in Mwanza
City. The current study did not observed E. coli ST-131 from fish however it was obtained from environment; this could be due to that only 2 out 4 isolates from fish were sequenced. Further studies with large number of $E$. coli from fish are needed to confirm the role of food chain in the persistence of bla $a_{\mathrm{CTX}-\mathrm{M}-15}$ in Mwanza city.

\section{CONCLUSION}

This is the first report on the epidemiology of ESBL-producing Gram-negative bacteria from fish in Lake Victoria and its surrounding environment. More than $70 \%$ of the sequenced bacterial isolates carried quinolone and aminoglycoside resistance genes. Detection of isolates/plasmids carrying bla CTX-M-15 which have been also found in humans and 
companion/livestock animals in the same region suggest environmental contamination with sewage effluents from humans an animal sources. Our data suggest that additional efforts to implement better sanitation and control sewage management are warranted. Many common illnesses in particular diarrhea, can be attributed to poor sanitation and unsafe water (Cheng et al., 2005) which exacerbates antibiotic use and contributes significantly to the problem of antibiotic resistance. Policy measures to improve water and sanitation quality could greatly contribute to the reduction of ESBL-resistance in this region.

\section{AUTHOR CONTRIBUTIONS}

NM, MM, CI, TC, and SM conceived, designed and executed the study; EM, NM, and SM collected the data and samples; NM, EM, and SM performed laboratory analysis; LF and TC performed WGS; LF, HG, TC, and SM analyzed the data; NM, LF, TC, and SM wrote the manuscript which was critically reviewed by all authors. All authors have read and approved the final draft of the manuscript.

\section{REFERENCES}

Abgottspon, H., Nüesch-Inderbinen, M. T., Zurfluh, K., Althaus, D., Hächler, H., and Stephan, R. (2014). Enterobacteriaceae with extended-spectrum-and pAmpC-type $\beta$-lactamase-encoding genes isolated from freshwater fish from two lakes in Switzerland. Antimicrob. Agents Chemother. 58, 2482-2484. doi: 10.1128/AAC.02689-13

Ahmed, A. M., and Shimamoto, T. (2008). Emergence of a cefepimeand cefpirome-resistant Citrobacter freundii clinical isolate harbouring a novel chromosomally encoded AmpC $\beta$-lactamase, CMY-37. Int. J. Antimicrob. Agents 32, 256-261. doi: 10.1016/j.ijantimicag.2008. 04.019

Ahmed, S. A., Awosika, J., Baldwin, C., Bishop-Lilly, K. A., Biswas, B., Broomall, S., et al. (2012). Genomic comparison of Escherichia coli O104: H4 isolates from 2009 and 2011 reveals plasmid, and prophage heterogeneity, including shiga toxin encoding phage stx2. PLoS ONE 7:e48228. doi: 10.1371/journal.pone.0048228

Alikhan, N. F., Petty, N. K., Zakour, N. L. B., and Beatson, S. A. (2011). BLAST Ring Image Generator (BRIG): simple prokaryote genome comparisons. BMC Genomics 12:402. doi: 10.1186/1471-2164-12-402

Arpin, C., Dubois, V., Maugein, J., Jullin, J., Dutilh, B., Brochet, J.-P., et al. (2005). Clinical and molecular analysis of extended-spectrum $\beta$-lactamase-producing enterobacteria in the community setting. J. Clin. Microbiol. 43, 5048-5054. doi: 10.1128/JCM.43.10.5048-5054.2005

Bankevich, A., Nurk, S., Antipov, D., Gurevich, A. A., Dvorkin, M., Kulikov, A. S., et al. (2012). SPAdes: a new genome assembly algorithm and its applications to single-cell sequencing. J. Comput. Biol. 19, 455-477. doi: $10.1089 / \mathrm{cmb} .2012 .0021$

Beninati, C., Reich, F., Muscolino, D., Giarratana, F., Panebianco, A., Klein, G., et al. (2015). ESBL-producing bacteria and MRSA isolated from poultry and Turkey products imported from Italy. Czech J. Food Sci. 33, 97-102. doi: 10.17221/428/2014-CJFS

Blaak, H., de Kruijf, P., Hamidjaja, R. A., van Hoek, A. H., de Roda Husman, A. M., and Schets, F. M. (2014). Prevalence and characteristics of ESBLproducing $E$. coli in Dutch recreational waters influenced by wastewater treatment plants. Vet. Microbiol. 171, 448-459. doi: 10.1016/j.vetmic.2014. 03.007

Carattoli, A., Zankari, E., García-Fernández, A., Larsen, M., Lund, O., Villa, L., et al. (2014). In silico detection and typing of plasmids using PlasmidFinder and plasmid multilocus sequence typing. Antimicrob. Agents Chemother. 58, 3895-3903. doi: 10.1128/AAC.02412-14

\section{ACKNOWLEDGMENTS}

We are grateful to all of the fish vendors and fishermen who helped us to get useful information during sample collection. Our heartfelt thanks also go to Mr. Vitus Silago for the diligent technical laboratory assistance he provided during the study period. We thank Christina Gerstmann from the Institute of Medical Microbiology in Germany for excellent technical assistance. This study was supported by a grant from the Wellcome Trust (WT087546MA) to SACIDS and also by grants from the Bundesministerium fuer Bildung und Forschung (BMBF, Germany) within the German Center for Infection Research [DZIF/grant number 8000 701-3 (HZI) to CI and TC; TI06.001/8032808811 to TC] and the research network RESET (grant number $01 \mathrm{KI} 1313 \mathrm{G}$ to CI and TC).

\section{SUPPLEMENTARY MATERIAL}

The Supplementary Material for this article can be found online at: http://journal.frontiersin.org/article/10.3389/fmicb. 2016.01862/full\#supplementary-material

Cheng, A. C., Mcdonald, J. R., and Thielman, N. M. (2005). Infectious diarrhea in developed and developing countries. J. Clin. Gastroenterol. 39, 757-773. doi: 10.1097/01.mcg.0000177231.13770.07

Clermont, O., Bonacorsi, S., Bingen, E., and Bonacorsi, P. (2000). Rapid and simple determination of the Escherichia coli phylogenetic. Appl. Environ. Microbiol. 66, 4555-4558. doi: 10.1128/AEM.66.10.4555-4558.2000

Dobiasova, H., Videnska, P., and Dolejska, M. (2015). Complete sequences of IncU plasmids harboring quinolone resistance genes qnrS2 and $a a c\left(6^{\prime}\right)-I b-c r$ in Aeromonas spp. from ornamental fish. Antimicrob. Agents Chemother. 60, 653-657. doi: 10.1128/AAC.01773-15

Dos Santos, G., Solidônio, E., Costa, M., Melo, R., De Souza, I., Silva, G., et al. (2015). "Study of the Enterobacteriaceae group CESP (Citrobacter, Enterobacter, Serratia, Providencia, Morganella and Hafnia): a review," in The Battle Against Microbial Pathogens: Basic Science, Technological Advances and Educational Programs, ed A. Méndez-Vilas (Badajoz: Formatex), 794-805.

Falgenhauer, L., Yao, Y., Fritzenwanker, M., Schmiedel, J., Imirzalioglu, C., and Chakraborty, T. (2014). Complete genome sequence of phage-like plasmid pECOH89, Encoding CTX-M-15. Genome Announc. 2:e00356-14. doi: 10.1128/genomea.00356-14

Fitzpatrick, M., Butler, S., Ham, A., and Hardy, P. (2015). Lonely Planet Tanzania Travel Guide. Oakland, CA: Amazon.

Fortini, D., Fashae, K., Villa, L., Feudi, C., García-Fernández, A., and Carattoli, A. (2015). A novel plasmid carrying bla $a_{\mathrm{CTX}-\mathrm{M}-15}$ identified in commensal Escherichia coli from healthy pregnant women in Ibadan, Nigeria. J. Glob. Antimicrob. Resist. 3, 9-12. doi: 10.1016/j.jgar.2014.12.002

Guenther, S., Ewers, C., and Wieler, L. H. (2011). Extended-spectrum betalactamases producing $E$. coli in wildlife, yet another form of environmental pollution? Front. Microbiol. 2:246. doi: 10.3389/fmicb.2011.00246

Hawkey, P. M., and Jones, A. M. (2009). The changing epidemiology of resistance. J. Antimicrob. Chemother. 64, i3-i10. doi: 10.1093/jac/dkp256

Ho, P.-L., Chow, K., Lai, E. L., Lo, W. U., Yeung, M., Chan, J., et al. (2011). Extensive dissemination of CTX-M-producing Escherichia coli with multidrug resistance to 'critically important' antibiotics among food animals in Hong Kong, 2008-10. J. Antimicrob. Chemother. 66, 765-768. doi: 10.1093/jac/dkq539

Ibrahim, H. M. M., Ahmed, A. M., El-Seedy, Y. Y., and El-Khodery, S. A. (2015). Distribution of multidrug-resistant gram negative bacteria causing clinical mastitis in dairy Cows. Glob. Vet. 15, 268-277. doi: 10.5829/idosi.gv.2015.15.03.1012

Ishida, Y., Ahmed, A. M., Mahfouz, N. B., Kimura, T., El-Khodery, S. A., Moawad, A. A., et al. (2010). Molecular analysis of antimicrobial resistance in 
Gram-negative bacteria isolated from fish farms in Egypt. J. Vet. Med. Sci. 72, 727-734. doi: 10.1292/jvms.09-0538

Jiang, H.-X., Tang, D., Liu, Y.-H., Zhang, X.-H., Zeng, Z.-L., Xu, L., et al. (2012). Prevalence and characteristics of $\beta$-lactamase and plasmid-mediated quinolone resistance genes in Escherichia coli isolated from farmed fish in China. J. Antimicrob. Chemother. 67, 2350-2353. doi: 10.1093/jac/dks250

Kayange, N., Kamugisha, E., Mwizamholya, D. L., Jeremiah, S., and Mshana, S. E. (2010). Predictors of positive blood culture and deaths among neonates with suspected neonatal sepsis in a tertiary hospital, Mwanza-Tanzania. $B M C$ Pediatr. 10:39. doi: 10.1186/1471-2431-10-39

Kümmerer, K. (2009). Antibiotics in the aquatic environment-a review-part I. Chemosphere 75, 417-434. doi: 10.1016/j.chemosphere.2008.11.086

Larsen, M. V., Cosentino, S., Rasmussen, S., Friis, C., Hasman, H., Marvig, R. L., et al. (2012). Multilocus sequence typing of total-genome-sequenced bacteria. J. Clin. Microbiol. 50, 1355-1361. doi: 10.1128/JCM.06094-11

Laube, H., Friese, A., von Salviati, C., Guerra, B., Käsbohrer, A., Kreienbrock, L., et al. (2013). Longitudinal monitoring of extended-spectrumbeta-lactamase/AmpC-producing Escherichia coli at German broiler chicken fattening farms. Appl. Environ. Microbiol. 79, 4815-4820. doi: 10.1128/AEM.00856-13

Liu, B. T., Liao, X. P., Yang, S. S., Wang, X. M., Li, L. L., Sun, J., et al. (2012). Detection of mutations in the gyrA and parC genes in Escherichia coli isolates carrying plasmid-mediated quinolone resistance genes from diseased food-producing animals. J. Med. Microbiol. 61, 1591-1599. doi: 10.1099/jmm.0.043307-0

Madec, J.-Y., Poirel, L., Saras, E., Gourguechon, A., Girlich, D., Nordmann, P., et al. (2012). Non-ST131 Escherichia coli from cattle harbouring humanlike blaCTX-M-15-carrying plasmids. J. Antimicrob. Chemother. 67, 578-581. doi: $10.1093 / \mathrm{jac} / \mathrm{dkr} 542$

Martinez, J. L. (2009a). Environmental pollution by antibiotics and by antibiotic resistance determinants. Environ. Pollut. 157, 2893-2902. doi: 10.1016/j.envpol.2009.05.051

Martinez, J. L. (2009b). The role of natural environments in the evolution of resistance traits in pathogenic bacteria. Proc. R. Soc. Lond. B Biol. Sci. 276, 2521-2530. doi: $10.1098 / \mathrm{rspb} .2009 .0320$

Moremi, N., Mushi, M. F., Fidelis, M., Chalya, P., Mirambo, M., and Mshana, S. E. (2014). Predominance of multi-resistant gram-negative bacteria colonizing chronic lower limb ulcers (CLLUs) at Bugando Medical Center. BMC Res. Notes 7:211. doi: 10.1186/1756-0500-7-211

Mshana, S. E., Falgenhauer, L., Mirambo, M. M., Mushi, M. F., Moremi, N., Julius, R., et al. (2016). Predictors of bl a CTX-M-15 in varieties of Escherichia coli genotypes from humans in community settings in Mwanza, Tanzania. BMC Infect. Dis. 16:187. doi: 10.1186/s12879-016-1527-x

Mshana, S. E., Hain, T., Domann, E., Lyamuya, E. F., Chakraborty, T., and Imirzalioglu, C. (2013). Predominance of Klebsiella pneumoniae ST14 carrying CTX-M-15 causing neonatal sepsis in Tanzania. BMC Infect. Dis. 13:466. doi: 10.1186/1471-2334-13-466

Mshana, S. E., Kamugisha, E., Mirambo, M., Chakraborty, T., and Lyamuya, E. F. (2009). Prevalence of multiresistant gram-negative organisms in a tertiary hospital in Mwanza, Tanzania. BMC Res. Notes 2:49. doi: 10.1186/1756-0500-2-49

Mshana, S., Imirzalioglu, C., Hain, T., Domann, E., Lyamuya, E. F., and Chakraborty, T. (2011). Multiple ST clonal complexes, with a predominance of ST131, of Escherichia coli harbouring blaCTX-M-15 in a tertiary hospital in Tanzania. Clin. Microbiol. Infect. 17, 1279-1282. doi: 10.1111/j.1469-0691.2011.03518.x

Murray, P. R., Baron, E. J., Pfaller, M., Tenover, F., Yolken, R. H., and Morgan, D. R. (1995). Manual of clinical microbiology (6th edn). Trends Microbiol. 3, $449-449$.

Pitout, J. D., Nordmann, P., Laupland, K. B., and Poirel, L. (2005). Emergence of Enterobacteriaceae producing extended-spectrum $\beta$-lactamases (ESBLs) in the community. J. Antimicrob. Chemother. 56, 52-59. doi: 10.1093/jac/ dki166

Rafaï, C., Frank, T., Manirakiza, A., Gaudeuille, A., Mbecko, J. R., Nghario, L., et al. (2015). Dissemination of IncF-type plasmids in multiresistant CTX-M-15-producing Enterobacteriaceae isolates from surgical-site infections in Bangui, Central African Republic. BMC Microbiol. 15:15. doi: 10.1186/s12866-015-0348-1

Rissman, A. I., Mau, B., Biehl, B. S., Darling, A. E., Glasner, J. D., and Perna, N. T. (2009). Reordering contigs of draft genomes using the Mauve aligner. Bioinformatics 25, 2071-2073. doi: 10.1093/bioinformatics/btp356

Seni, J., Falgenhauer, L., Simeo, N., Mirambo, M. M., Imirzalioglu, C., Matee, M., et al. (2016). Multiple ESBL-producing Escherichia coli sequence types carrying quinolone and aminoglycoside resistance genes circulating in companion and domestic farm animals in Mwanza, Tanzania, harbor commonly occurring plasmids Front. Microbiol. 7:142. doi: 10.3389/fmicb.2016. 00142

Treangen, T. J., Ondov, B. D., Koren, S., and Phillippy, A. M. (2014). The Harvest suite for rapid core-genome alignment and visualization of thousands of intraspecific microbial genomes. Genome Biol. 15:524. doi: 10.1186/s13059-014-0524-x

van Hoek, A. H., Veenman, C., van Overbeek, W. M., Lynch, G., de Roda Husman, A. M., and Blaak, H. (2015). Prevalence and characterization of ESBL-and AmpC-producing Enterobacteriaceae on retail vegetables. Int. J. Food Microbiol. 204, 1-8. doi: 10.1016/j.ijfoodmicro.2015.03.014

Wayne, P. (2012). Performance Standards for Antimicrobial Susceptibility Testing: Twenty-Second Informational Supplement. Clinical and Laboratory Standards Institute. M100-S22. USA.

Weigel, L. M., Steward, C. D., and Tenover, F. C. (1998). gyrA mutations associated with fluoroquinolone resistance in eight species of Enterobacteriaceae. Antimicrob. Agents Chemother. 42, 2661-2667.

Welch, T. J., Evenhuis, J., White, D. G., Mcdermott, P. F., Harbottle, H., Miller, R. A., et al. (2009). IncA/C plasmid-mediated florfenicol resistance in the catfish pathogen Edwardsiella ictaluri. Antimicrob. Agents Chemother. 53, 845-846. doi: 10.1128/AAC.01312-08

Xi, M., Wu, Q., Wang, X., Yang, B., Xia, X., and Li, D. (2015). Characterization of extended-spectrum $\beta$-lactamase-producing Escherichia coli strains isolated from retail foods in Shaanxi Province, China. J. Food Prot.78, 1018-1023. doi: 10.4315/0362-028X.JFP-14-490

Zankari, E., Hasman, H., Cosentino, S., Vestergaard, M., Rasmussen, S., Lund, O., et al. (2012). Identification of acquired antimicrobial resistance genes. J. Antimicrob. Chemother. 67, 2640-2644. doi: 10.1093/jac/ dks261

Zhang, L., Lü, X., and Zong, Z. (2013). The emergence of blaCTX-M-15-carrying Escherichia coli of ST131 and new sequence types in Western China. Ann. Clin. Microbiol. Antimicrob. 12:35. doi: 10.1186/1476-0711-12-35

Zurfluh, K., Hächler, H., Nüesch-Inderbinen, M., and Stephan, R. (2013). Characteristics of extended-spectrum $\beta$-lactamase-and carbapenemaseproducing Enterobacteriaceae isolates from rivers and lakes in Switzerland. Appl. Environ. Microbiol. 79, 3021-3026. doi: 10.1128/AEM.00054-13

Conflict of Interest Statement: The authors declare that the research was conducted in the absence of any commercial or financial relationships that could be construed as a potential conflict of interest.

Copyright (c) 2016 Moremi, Manda, Falgenhauer, Ghosh, Imirzalioglu, Matee, Chakraborty and Mshana. This is an open-access article distributed under the terms of the Creative Commons Attribution License (CC BY). The use, distribution or reproduction in other forums is permitted, provided the original author(s) or licensor are credited and that the original publication in this journal is cited, in accordance with accepted academic practice. No use, distribution or reproduction is permitted which does not comply with these terms. 